\title{
Must be $\Psi$ single-valued? Ring vs. periodic boundary conditions, spinors and double rotations
}

\author{
Josep Planelles* \\ Dept. Química-Física i Analítica, Universitat Jaume I
}

January 5, 2022

\begin{abstract}
This is a lecture notes for undergraduate students. We try to tackle the single valuedness of spatial and double valuedness of spin functions. Also, we adress the need of spinors to accommodate spin functions with some parallelism to the need of axial vectors (or antisymmetric traceless tensors) to accommodate angular momentum. Finally, we revisit the Dirac and Weyl tricks on the non-equivalence of a $2 \pi$ and a $4 \pi$ rotation related the topology of rotation and unitary groups.
\end{abstract}

\section{Introduction}

The requirement that wave function in non-relativistic quantum mechanics must be single valued is sometimes assumed as a postulate (see e.g. McWeeny[1]). However, since the wave function $\Psi$ is not an observable by itself but by the square of its modulus $|\Psi|^{2}$, it seems sensible to require single valuedness to $|\Psi|^{2}$ instead.

As a matter of fact, the boundary conditions in periodic systems' band calculation come from the assumption that the crystal is invariant under translations, i.e., that there is no observable difference if an electron is located at $x$ or at $x+a$, with $a$ being the cell constant. Therefore, we set $|\Psi(x)|^{2}=|\Psi(x+a)|^{2}$ and it follows $\Psi(x+a)=e^{i \phi} \Psi(x)$, i.e., the wave function at $x$ and $x+a$ are identical up to a phase. Then, we solve the Schrödinger equation for every phase $e^{i \phi}, \phi \in[-\pi, \pi]$. The plot of the obtained energies vs. $\phi$ is a set of continuous energy bands separated by forbidden gaps originated from Bragg diffraction at $2 a=n \lambda, n=1,2, \ldots$ with $\lambda$ being DeBroglie wavelength of the electron.

In a similar way, when a particle is freely traveling in a radius $R$ ring, its position fully fixed by an angle $\theta$, a full rotation from $\theta$ up to $\theta+2 \pi$ brings the system to a new angle without observable changes. Then, we may say that, similar to the periodic crystal where $x$ is equivalent to $x+a$, also in the ring $\theta$ should be equivalent to $\theta+2 \pi$. Then, it follows $|\Psi(\theta)|^{2}=|\Psi(\theta+2 \pi)|^{2}$, i.e., $\Psi(\theta+2 \pi)=e^{i \phi} \Psi(\theta)$, and we would proceed to solve the Schrödinger equation for every phase $\left\{e^{i \phi}, \phi \in[-\pi, \pi]\right\}$. As a result, an energy continuum would be reached instead of the well-known quantification of the angular momentum.

What is the difference between both cases? Locally, the particle feels equivalent environments on the straight line $\mathbb{R}$ and on the ring $S^{1}$. As a matter of fact, a unit radius $S^{1}$ circle can be thought as the $[0,2 \pi]$ interval in $\mathbb{R}$ with the interval extremes identified and the whole real line rolled up (see figure), so that translations on the line are identified with rotations along this circumference.

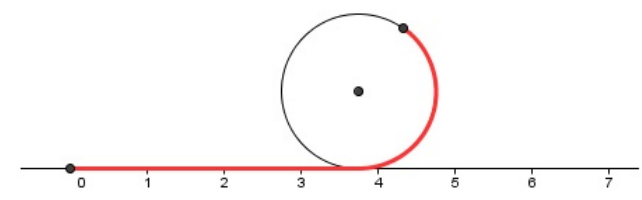

*email: josep.planelles@uji.es 
However, if we see the $\mathbb{R}$ line and the $S^{1}$ ring embedded in $\mathbb{R}^{2}$ we observe global differences. For example, we can define circumference's interior and exterior, while cannot do it for the line. It provides $S^{1}$ and $\mathbb{R}$ with different topology. Other differences arise if we consider an infinite ribbon with thickness instead of just a straight line and a small cylinder instead of a ring: the ribbon is simply connected while the cylinder is doubly connected. Furthermore, should we consider the ring and the periodic crystal embedded in $\mathbb{R}^{3}$ we find that ring positions $\theta$ and $\theta+2 \pi$ label the same point of $\mathbb{R}^{3}$ while $x$ i $x+a$ on the periodic line do not. Deep differences between rotations and translations derive from the fact that paths on a plane are commutative while are not on a sphere. Translation commutativity originates on the commutation of any two components of the linear momentum (which are generators of translation) while non-commutativity of rotations is a consequence of the non-commutativity of angular momentum components (rotation's generators).

In short, $x$ and $x+a$ are equivalent but not the same point, so we say $|\Psi(x)|^{2}=|\Psi(x+a)|^{2}$. On the other hand, $\theta$ and $\theta+2 \pi$ are actually the same point and so, in order to avoid having a multivalued functions, we say $\Psi(\theta+2 \pi)=\Psi(\theta)$. A question arises: Is single valuedness an additional postulate of quantum mechanics or is it a consequence of formalism itself? A second question emerges: what about spin functions?

Within non-relativistic quantum mechanics without spin, the use of ladder operators, defined from the angular momentum commutation relations, leads to integer or semi-integer values for the angular momentum quantum number $\ell$. The analytic calculation of eigenfunctions for integer values of $\ell$ yields the spherical harmonics which are single valued functions of the particle position. Fractional quantum numbers leads the wave function to change its sign under a $2 \pi$ rotation of the axial angle $\phi$, i.e., is a double-valued function. As we will see later, spherical harmonics with fractional values of $\ell$ have undesirable properties and, in fact, do not constitute any basis set for the three-dimensional rotations $S O(3)$ symmetry group representations. As Merzbacher reminds, [2] Pauli suggested replacing the postulate of single valuedness by the requirement that functions must build basis for the symmetry group representations (see appendix). A similar reasoning can be found in Le Bellac.[3] On the other hand, Riess claims that it is not necessary to impose the single valuedness character on non-relativistic wave functions without spin, because he showed[4] it derives from the elliptic character[5] of the Schrödinger equation. At any rate, as Merzbacher points out,[2] the single valuedness condition is deeply rooted in the foundations of quantum mechanics and it is not unreasonable, as McWeeny does, [1] to simplify the discussion and include it as an additional postulate.

These considerations do not affect spin functions, for they are not a function of spatial coordinates. Therefore, we must tackle the meaning of the sentence it requires a double rotation ( $4 \pi$ ) to return to the initial position. It suggests the spin as living on a Möbius strip embedded in $\mathbb{R}^{3}$. In fact, Ho and Morgan [6] show that the orbital angular momentum can assume half-integer values in Kaluza-Klein's multiply connected topological space (that suggests an identification with spin).

\section{Spin}

Stern and Gerlach's experiment tells us that electron has an intrinsic angular momentum, we call it spin $S$, with modulus $\frac{3}{4} \hbar^{2}$ and two possible orientations $S_{z}= \pm \frac{1}{2} \hbar$. The angular momentum eigenvalue equations in spherical coordinates,

$$
\begin{aligned}
& \hat{L}_{z} Y_{\ell, m}(\theta, \phi)=-i \hbar \frac{\partial}{\partial \phi} Y_{\ell, m}(\theta, \phi)=m \hbar Y_{\ell, m}(\theta, \phi) \\
& \widehat{L^{2}} Y_{\ell, m}(\theta, \phi)=-\hbar^{2}\left[\frac{1}{\sin ^{2} \theta} \frac{\partial^{2}}{\partial \phi^{2}}+\frac{1}{\sin \theta} \frac{\partial}{\partial \theta}\left(\sin \theta \frac{\partial}{\partial \theta}\right)\right] Y_{\ell, m}(\theta, \phi)=\ell(\ell+1) \hbar^{2} Y_{\ell, m}(\theta, \phi)
\end{aligned}
$$

has $\Psi=N \sin ^{1 / 2} \theta e^{i \phi / 2}$ as a solution. Certainly, by injecting it into eq. (1a) we get $m=\frac{1}{2}$ while in (1b) 
it yields $\ell=\frac{1}{2}$. Namely, $\Psi$ is the spherical harmonic $Y_{\frac{1}{2}}, \frac{1}{2}$.

We can determine $Y_{\frac{1}{2},-\frac{1}{2}}$ by applying the ladder down operator $\hat{L}_{-}=\hat{L}_{x}-i \hat{L}_{y}$ on the above function.[7] In spherical coordinates this operator reads $[8] \hat{L}_{-}=\hbar e^{-i \phi}\left(-\frac{\partial}{\partial \theta}+i \cot \theta \frac{\partial}{\partial \phi}\right)$, and applied to $Y_{\frac{1}{2}, \frac{1}{2}}$ yields $\Psi_{2}=e^{-i \phi / 2} \cot \theta \sqrt{\sin \theta}$, which is not an allowed wavefunction (as it goes to infinity at $\theta=0$ and $\theta=\pi$ ), instead of yielding $\Psi_{2}=N \sin ^{1 / 2} \theta e^{-i \phi / 2}$, which is free from singularities and fulfills eqs. (1) for $\ell=\frac{1}{2}$, $m=-\frac{1}{2}$.

Another solution of the equations (1) is $Y_{\frac{3}{2}}, \frac{1}{2}=\sin ^{1 / 2} \theta \cos \theta e^{i \phi / 2}$, as can be easily checked. However, when calculating $\left\langle Y_{\frac{3}{2}, \frac{1}{2}}\left|\hat{L}_{x}\right| Y_{\frac{1}{2}, \frac{1}{2}}\right\rangle$ it results non-zero. It means that $\hat{L}_{x}$ mixes states with different quantum number $\ell$, and therefore means that $\hat{L}_{x}$ and $\widehat{L^{2}}$ do not commute with these functions.

On the other hand, the above $\theta, \phi$ variables should correspond to non-spatial but internal electron coordinates. Otherwise, we would employ the same operator for orbital and spin angular momentum which yields contradictory results, as for example that $\left[\hat{L}_{x}, \hat{S}_{y}\right]$ is non-zero as it must be.

In addition, the misbehavior of spherical harmonics with fractional $\ell$ excludes the possibility that $\hat{L}_{i}$ and $\hat{S}_{i}$ have the same shape but with different and independent coordinates. Therefore, in order to build up the formalism of spin functions we must start from solid evidences: the angular momentum modulus is $\frac{3}{4} \hbar^{2}$ and the $S_{z}$ components $\pm \frac{1}{2} \hbar$. As a result, we have a two-dimensional $\{\alpha, \beta\}$ basis set of $\widehat{S^{2}}, \hat{S}_{z}$ eigenfunctions corresponding to $s=\frac{1}{2}, m_{s}= \pm \frac{1}{2}$. At this stage, we do not know their analytic expression so we just represent them by the orthogonal column vectors $\alpha \equiv\left(\begin{array}{l}1 \\ 0\end{array}\right)$ and $\beta \equiv\left(\begin{array}{l}0 \\ 1\end{array}\right)$, that we assume normalized $(\langle\alpha \mid \alpha\rangle=\langle\beta \mid \beta\rangle=1,\langle\alpha \mid \beta\rangle=0)$. Any other normalized spin state, $\left(\begin{array}{l}a \\ b\end{array}\right)$ with $|a|^{2}+|b|^{2}=1$, is a linear combination of this basis.

Neither we know the form of the spin operators, but we can easily write the $\hat{S}_{z}$ matrix representation in this basis: it must be diagonal with the eigenvalues as diagonal elements:

$$
\mathbb{S}_{z}=\frac{\hbar}{2}\left(\begin{array}{cc}
1 & 0 \\
0 & -1
\end{array}\right) \equiv \frac{\hbar}{2} \sigma_{z}
$$

Next, we can use ladder operators, $\hat{S}_{ \pm}\left|s, m_{s}\right\rangle=\sqrt{s(s+1)-m_{s}\left(m_{s} \pm 1\right)} \hbar\left|s, m_{s} \pm 1\right\rangle$ to find out that $\hat{S}_{+} \alpha=\hat{S}_{-} \beta=0, \hat{S}_{+} \beta=\alpha, \hat{S}_{-} \alpha=\beta$. Then, the ladder operators matrix representation should be:

$$
\mathbb{S}_{+}=\hbar\left(\begin{array}{ll}
0 & 1 \\
0 & 0
\end{array}\right) \quad \mathbb{S}_{-}=\hbar\left(\begin{array}{ll}
0 & 0 \\
1 & 0
\end{array}\right)
$$

Finally,

$$
\mathbb{S}_{x}=\frac{1}{2}\left(\mathbb{S}_{+}+\mathbb{S}_{-}\right)=\frac{\hbar}{2}\left(\begin{array}{ll}
0 & 1 \\
1 & 0
\end{array}\right) \equiv \frac{\hbar}{2} \sigma_{x} \quad \mathbb{S}_{y}=\frac{1}{2 i}\left(\mathbb{S}_{+}-\mathbb{S}_{-}\right)=\frac{\hbar}{2}\left(\begin{array}{cc}
0 & -i \\
i & 0
\end{array}\right) \equiv \frac{\hbar}{2} \sigma_{y}
$$

It is straightforward to check the (correct) commutation of these matrices, e.g. $\left[\mathbb{S}_{x}, \mathbb{S}_{y}\right]=i \hbar \mathbb{S}_{z}$.

A characteristic feature of $Y_{\frac{1}{2}, \frac{1}{2}}=N \sin ^{1 / 2} \theta e^{i \phi / 2}$ is its double-valuedness character, i.e., $Y_{\frac{1}{2}, \frac{1}{2}}(\theta, \phi) \neq$ $Y_{\frac{1}{2}, \frac{1}{2}}(\theta, \phi+2 \pi)$ (and therefore the need of two complete turns to recover the starting point). We wonder if this is a feature of these misbehaving functions or if the spin functions must actually be multivalued.

In ordinary space rotations of functions are generated by the angular momentum: $R_{\mathbf{u}}(\theta)=e^{i \frac{\theta}{\hbar} \mathbf{L} \cdot \mathbf{u}}$. However, orbital angular momentum does not act on the spin functions. Therefore, it is to be expected that rotations of spin functions will be generated by the spin angular momentum. We write each component: 


$$
\begin{aligned}
& R_{z}(\theta)=e^{i \frac{\theta}{\hbar} S_{z}}=e^{i \frac{\theta}{2} \sigma_{z}} ; \operatorname{amb} \sigma_{z}=\left(\begin{array}{cc}
1 & 0 \\
0 & -1
\end{array}\right) \\
& R_{x}(\theta)=e^{i \frac{\theta}{\hbar} S_{x}}=e^{i \frac{\theta}{2} \sigma_{x}} ; \operatorname{amb} \sigma_{x}=\left(\begin{array}{cc}
0 & 1 \\
1 & 0
\end{array}\right) \\
& R_{y}(\theta)=e^{i \frac{\theta}{\hbar} S_{y}}=e^{i \frac{\theta}{2} \sigma_{y}} ; \operatorname{amb} \sigma_{y}=\left(\begin{array}{cc}
0 & -i \\
i & 0
\end{array}\right)
\end{aligned}
$$

Half angles in the rotation operators, which come from the fractional value of the spin quantum number $s=\frac{1}{2}$, require a $4 \pi$ rotation to recover the identity matrix. In this sense, spin functions, hereafter spinors, must rotate $4 \pi$ to recover the original position. The spinor is like a vector on a Möbius strip, where you have to roll the tape twice to return to the initial position.

\section{Spinors}

Mass and electric charge are scalar, i.e., they are invariant under rotations. Linear momentum is not, but is transformed by rotations in a characteristic way we call vectorial. We say that linear momentum is a three coordinates' vector. Quadrupole moment or inertia tensor transformations under rotations requires a generalization of vector's concept. We say they are second-order tensors and are represented by a sixindependent coordinates symmetric $3 \times 3$ matrix. The angular momentum or magnetic field, which we usually represent as a three-coordinates vectors, behave under rotations like the linear momentum and in this sense they look like a vector, but are transformed differently under reflections. And they are said to be axial vectors, which is a way of hiding its three-independent-coordinates antisymmetric zero-trace tensor character. ${ }^{1}$ In order to accommodate e.g. the electric octupole moment or other physical quantities like it, we can define higher range tensors. In short, mass is a zero-range tensor, linear momentum is a 1-rank tensor, moment of inertia a 2-rank tensor, and so on. The spin functions, though, do not fit the tensor definition. We should generalize tensors to accommodate spin. Something similar to what happens to the magnetic field happens to the spinors: they look in various ways like vectors, but behaves differently under rotations. Next we will generalize the concept of vector to accommodates spin and will refer it to as 1-rank spinor or just spinor. Once defined 1-rank spinors, we define higher rank spinors in a way similar to that defining higher rank tensors from vectors (or 1-rank tensor).

We can visualize an spinor (see figure) as the positional state of a rotating symmetric top: angles $\theta$ and $\phi$ determine the rotation axis, the vector length $r$ along this axis is set to the rotation speed, $\alpha$ is the rotation angle, and a sign must be supplied to represent the rotation sense. Therefore, disregarding for the time being the sign which we will consider positive unless otherwise is said, we can represent an spinor by 4 real numbers. ${ }^{2}$

${ }^{1}$ The reflection plane $\sigma_{x y}$, which we may represent by the matrix $\sigma_{x y}=\left(\begin{array}{ccc}1 & 0 & 0 \\ 0 & 1 & 0 \\ 0 & 0 & -1\end{array}\right)$, acts on a $\mathbb{R}^{3}$ vector by changing the sign of its third coordinate. Therefore, if we represent the magnetic field with a $\mathbb{R}^{3}$ vector and apply $\sigma_{x y}$ on it we get a wrong result. But if we represent it by the antisymmetric tensor $\mathbb{B}=\left(\begin{array}{ccc}0 & z & y \\ -z & 0 & x \\ -y & -x & 0\end{array}\right)$, the corresponding transformation, $\mathbb{B}^{\prime}=\sigma_{x y}^{t} \mathbb{B} \sigma_{x y}=\mathbb{B}$, behaves as it must.

${ }^{2}$ In fact, an equivalent way of defining spinors is to consider them as four-dimensional objects in space-time that undergo Lorentz transformations.[9] 


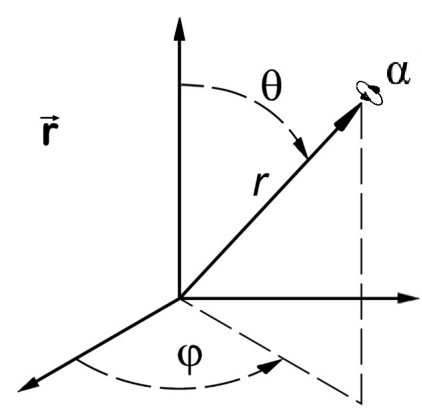

Alternatively, since we have represented the $\alpha, \beta$ spin functions by two-row column arrays, we can represent the spinor by two complex numbers $z_{1}=r_{1} e^{i \theta_{1}}, z_{2}=r_{2} e^{i \theta_{2}}$. From them we can extract four real numbers as follows:

$$
\begin{aligned}
& p_{1}=\left|z_{1}\right|^{2}+\left|z_{1}\right|^{2}=r_{1}^{2}+r_{2}^{2} \\
& p_{2}=\left|z_{1}\right|^{2}-\left|z_{1}\right|^{2}=r_{1}^{2}-r_{2}^{2} \\
& p_{3}=z_{1} z_{2}^{*}+z_{1}^{*} z_{2}=r_{1} r_{2}\left(e^{i\left(\theta_{1}-\theta_{2}\right)}+e^{-i\left(\theta_{1}-\theta_{2}\right)}\right)=r_{1} r_{2} 2 \cos \Delta \theta \\
& p_{4}=i\left(z_{1} z_{2}^{*}-z_{1}^{*} z_{2}\right)=i r_{1} r_{2}\left(e^{i\left(\theta_{1}-\theta_{2}\right)}-e^{-i\left(\theta_{1}-\theta_{2}\right)}\right)=r_{1} r_{2}(-2) \sin \Delta \theta
\end{aligned}
$$

We can identify these real numbers with the module and the components of the vector $\mathbf{r}$ that describes the rotation axis and speed if we write $r_{1}=\sqrt{r} \cos \frac{\theta}{2}$ and $r_{2}=\sqrt{r} \sin \frac{\theta}{2}$. Then, it follows that $p_{1}=r_{1}^{2}+r_{2}^{2}=r$ and $p_{2}=r_{1}^{2}-r_{2}^{2}=r\left(\cos ^{2} \frac{\theta}{2}-\sin ^{2} \frac{\theta}{2}\right)=r \cos \theta=r_{z}$. Also, $r_{1} r_{2}=r \sin \frac{\theta}{2} \cos \frac{\theta}{2}=\frac{1}{2} r \sin \theta$. Therefore, we may identify $p_{3}=r_{1} r_{2} 2 \cos \Delta \theta=r \sin \theta \cos \Delta \theta$ with $r_{x}$ and $p_{4}=r_{1} r_{2}(-2) \sin \Delta \theta=r \sin \theta(-\sin \Delta \theta)$ with $r_{y}$ if $\Delta \theta=-\phi$, and to this we set $\theta_{1}=-\frac{\alpha+\phi}{2}$ and $\theta_{2}=\frac{\alpha-\phi}{2}$.

With these identifications we can write $z_{1}=\sqrt{r} \cos \frac{\theta}{2} e^{-i(\alpha+\phi) / 2}$ and $z_{2}=\sqrt{r} \sin \frac{\theta}{2} e^{-i(\alpha-\phi) / 2}$ and represent the spinor by means of the following complex vector:

$$
|s\rangle=\sqrt{r} e^{-i \alpha / 2}\left(\begin{array}{c}
\cos \frac{\theta}{2} e^{-i \phi / 2} \\
\sin \frac{\theta}{2} e^{i \phi / 2}
\end{array}\right)
$$

In particular, $r=1, \alpha=0, \theta=0, \phi=0$ yield the $\left(\begin{array}{l}1 \\ 0\end{array}\right)$ spinor , while $r=1, \alpha=0, \theta=\pi, \phi=0$ yield $\left(\begin{array}{l}0 \\ 1\end{array}\right)$. The r-vector coordinates $r_{x}, r_{y}, r_{z}$ can also be calculated from $|s\rangle$ with the help of Pauli's matrices as follows:

$$
r_{x}=\left\langle s\left|\sigma_{x}\right| s\right\rangle \quad r_{y}=\left\langle s\left|\sigma_{y}\right| s\right\rangle \quad r_{z}=\left\langle s\left|\sigma_{z}\right| s\right\rangle
$$

Let's check $r_{x}\left(r_{y}, r_{x}\right.$ can be checked in a similar way):

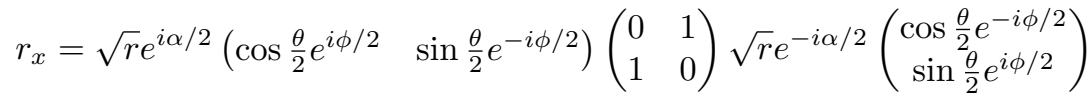

$$
\begin{aligned}
& =r\left(\cos \frac{\theta}{2} e^{i \phi / 2} \quad \sin \frac{\theta}{2} e^{-i \phi / 2}\right)\left(\begin{array}{c}
\sin \frac{\theta}{2} e^{i \phi / 2} \\
\cos \frac{\theta}{2} e^{-i \phi / 2}
\end{array}\right) \\
& =r \cos \frac{\theta}{2} \sin \frac{\theta}{2}\left(e^{i \phi}+e^{-i \phi}\right)=r \sin \theta \cos \phi .
\end{aligned}
$$




\section{Spinors' transformations}

Since we represent a spinor by a complex two-dimensional vector, the transformations that preserve the length of this vector are the $2 \times 2$ unitary transformations of unit determinant (these matrices are the elements of the so-called special unitary two-dimensional $S U(2)$ Lie grup). Indeed, from the definition of unitary matrix, $\mathbb{U}^{\dagger} \mathbb{U}=\mathbb{U}^{\dagger}=\mathbb{1}$, we can write $\mathbb{U}$ as the exponential of a hermitian matrix $\mathbb{A}$ (i.e. $\left.\mathbb{A}=\mathbb{A}^{\dagger}\right)$. We have then $\mathbb{U}=e^{i \mathbb{A}}, \mathbb{U}^{\dagger}=e^{-i \mathbb{A}}$ and so $\mathbb{U}^{\dagger}=e^{i \mathbb{A}} e^{-i \mathbb{A}}=\mathbb{1}$.

On the other hand, since $\mathbb{U} \mathbb{U}^{\dagger}=\mathbb{1}$, we have $\operatorname{det}(\mathbb{U}) \operatorname{det}\left(\mathbb{U}^{\dagger}\right)=\operatorname{det}(\mathbb{U}) \operatorname{det}(\mathbb{U})^{*}=1$. Therefore, $\operatorname{det}(\mathbb{U})$ must be a phase, i.e., a complex unit length number $e^{i \phi}$. Also, since the determinant of the exponential of a matrix is equal to the exponential of the matrix trace, $\operatorname{det}\left(e^{\mathbb{A}}\right)=e^{\operatorname{tr}(\mathbb{A})}$, a traceless hermitian matrix is required to generate a unit determinant unitary matrix $\mathbb{U}=e^{i \mathbb{A}}$.

We can always write a traceless hermitian matrix in the form:

$$
\left(\begin{array}{cc}
a & b-i c \\
b+i c & -a
\end{array}\right)=a\left(\begin{array}{cc}
1 & 0 \\
0 & -1
\end{array}\right)+b\left(\begin{array}{cc}
0 & 1 \\
1 & 0
\end{array}\right)+c\left(\begin{array}{cc}
0 & -i \\
i & 0
\end{array}\right)=a \sigma_{z}+b \sigma_{x}+c \sigma_{y}
$$

where, again, $\sigma_{i}$ are the Pauli's matrices. Therefore Pauli's matrices constitute the algebra of generators of unit determinant unitary transformations in two dimensions $S U(2) \operatorname{group:} \mathbb{U}=e^{i \theta \mathbf{n} \cdot \boldsymbol{\sigma}}$, with $\mathbf{n} \equiv|n\rangle$ being the unit vector along $\left(\begin{array}{lll}a & b & c\end{array}\right)^{\dagger}$ and $\theta$ representing its length. By drawing a parallel with the rotations in real three-dimensional spaces, which are generated by the angular momentum $\mathbb{R}(\theta)=e^{i \theta \mathbf{n} \cdot \mathbf{L}}$, we can see $\mathbb{U}$ as a rotation in a two-dimensional complex space.

\subsection{Spinors and vectors rotations}

In a previous section we have set the relationship between the spinor $|s\rangle=\sqrt{r} e^{-i \alpha / 2}\left(\begin{array}{c}\cos \frac{\theta}{2} e^{-i \phi / 2} \\ \sin \frac{\theta}{2} e^{i \phi / 2}\end{array}\right)$ and the polar vector $\mathbf{r}$ with cartesian coordinates $\left(r_{x}, r_{y}, r_{z}\right)$ (or spherical $(r, \theta, \phi)$ coordinates). In particular, we have seen that $r_{i}=\left\langle s\left|\sigma_{i}\right| s\right\rangle$, eq. (7), and have just shown that unitary transformations can be written as $\mathbb{U}=e^{i \theta \mathbf{n} \cdot \boldsymbol{\sigma}}$ and, by analogy with vector rotations in $\mathbb{R}^{3}$, we define three components $\mathbb{U}_{i}=e^{i \theta \sigma_{i}}$ with $i=x, y, z$. Next, we will show how spinor transformations translate into transformations of the associated $\mathbb{R}^{3}$ vector. To this end we recall here some identities that can be checked just by replacing symbols by matrices and carrying out matrix products: $\sigma_{z}^{2}=\sigma_{x}^{2}=\sigma_{y}^{2}=\mathbb{1}$. Then,

$$
e^{i \theta \sigma_{i}}=\sum_{n=0}^{\infty} \frac{(i \theta)^{n}}{n !} \sigma_{i}^{n}=\left(\sum_{0,2,4, \ldots}^{\infty} \frac{(i \theta)^{n}}{n !}\right) \mathbb{1}+\left(\sum_{1,3, \ldots}^{\infty} \frac{(i \theta)^{n}}{n !}\right) \sigma_{i}=\cos \theta \mathbb{1}+i \sin \theta \sigma_{i}
$$

as the first bracket is $\cos \theta$ Taylor's expansion and the second that of $\sin \theta$ times the imaginary number $i$.

Taking it into account, let's consider the spinor transformation $\mathbb{U}_{z}:\left|s^{\prime}\right\rangle=\mathbb{U}_{z}|s\rangle=e^{i \theta \sigma_{z}}|s\rangle$. And now let's calculate, by components, the associated vector transformation $\left|r^{\prime}\right\rangle=\left\langle s^{\prime}|\boldsymbol{\sigma}| s^{\prime}\right\rangle$ :

$$
\begin{aligned}
& z^{\prime}=\left\langle s^{\prime}\left|\sigma_{z}\right| s^{\prime}\right\rangle=\left\langle s\left|e^{-i \theta \sigma_{z}} \sigma_{z} e^{i \theta \sigma_{z}}\right| s\right\rangle=\left\langle s\left|\sigma_{z} e^{-i \theta \sigma_{z}} e^{i \theta \sigma_{z}}\right| s\right\rangle=\left\langle s\left|\sigma_{z}\right| s\right\rangle=z \\
& x^{\prime}=\left\langle s^{\prime}\left|\sigma_{x}\right| s^{\prime}\right\rangle=\left\langle s\left|\left(\cos \theta \mathbb{1}-i \sin \theta \sigma_{z}\right) \sigma_{x}\left(\cos \theta \mathbb{1}+i \sin \theta \sigma_{z}\right)\right| s\right\rangle
\end{aligned}
$$

At this point we recall some additional identities (that can also be verified by replacing symbols by matrices and carrying out matrix products): $\sigma_{x} \sigma_{y}=i \sigma_{z}, \sigma_{y} \sigma_{x}=-i \sigma_{z}$ and cyclic permutations. Then, $\sigma_{z} \sigma_{x} \sigma_{z}=-\sigma_{x}$, so:

$$
\begin{aligned}
x^{\prime} & =\left\langle s\left|\left(\cos ^{2} \theta \sigma_{x}+\sin ^{2} \theta \sigma_{z} \sigma_{x} \sigma_{z}+i \cos \theta \sin \theta \sigma_{x} \sigma_{z}-i \sin \theta \cos \theta \sigma_{z} \sigma_{x}\right)\right| s\right\rangle \\
& =\left\langle s\left|\sigma_{x}\right| s\right\rangle\left(\cos ^{2} \theta-\sin ^{2} \theta\right)+\left\langle s\left|\sigma_{y}\right| s\right\rangle 2 \sin \theta \cos \theta=x \cos 2 \theta+y \sin 2 \theta
\end{aligned}
$$


In a similar way, $y^{\prime}=-x \sin 2 \theta+y \cos 2 \theta$.

We see then that an spinor $\theta$ rotation translates into a $2 \theta$ rotation of the associated vector. In other words, a vector $2 \pi$ rotation is equivalent to a $\pi$ rotation of the spinor and, since $\mathbb{U}_{i}(\theta)=e^{i \theta \sigma_{i}}=\cos \theta \mathbb{1}+i \sin \theta \sigma_{i}$, then $\mathbb{U}_{i}(\pi)=-\mathbb{1}$, that means changing the spinor sign. It is needed a $4 \pi$ rotation for the spinor to recover the initial state. ${ }^{3}$

A consequence of the angle doubling in 3D rotations ( $S O(3)$ rotations group) with respect to complex $2 \mathrm{D}$ rotations $(S U(2)$ unitary group) is that every two $S U(2)$ elements correspond to a single $S O(3)$ element: the relationship $S U(2)-S O(3)$ is homomorphic but it is not isomorphic. In other words, spin functions form basis sets for $S O(3)$ projective representations, by projective representation meaning that the $R_{3}=R_{1} R_{2}$ matrix representation is $D\left(R_{3}\right)=\epsilon D\left(R_{1}\right) D\left(R_{2}\right)$ with $\epsilon \neq 1$ (while in ordinary representations, also called vector representations, $\epsilon=1)$.

\subsection{Higher rank spinors}

First, we will briefly show how to build up a rank 2 tensor $T_{x y}$ from two $\left|r_{1}\right\rangle,\left|r_{2}\right\rangle$ rank 1 tensors (also called polar vectors). We define $T_{x y}=\left|r_{1}\right\rangle\left\langle r_{2}\right|$. The corresponding matrix form is:

$$
T_{x y}=\left(\begin{array}{l}
x_{1} \\
y_{1} \\
z_{1}
\end{array}\right)\left(\begin{array}{lll}
x_{2} & y_{2} & z_{2}
\end{array}\right)=\left(\begin{array}{lll}
x_{1} x_{2} & x_{1} y_{2} & x_{1} z_{2} \\
y_{1} x_{2} & y_{1} y_{2} & y_{1} z_{2} \\
z_{1} x_{2} & z_{1} y_{2} & z_{1} z_{2}
\end{array}\right)
$$

Please note that should $\left|r_{1}\right\rangle=\left|r_{2}\right\rangle$ then the resulting tensor is symmetric. From a general rank 2 tensor we can extract a traceless symmetric, an anti-symmetric and an scalar tensors as follows:

$$
\begin{aligned}
\mathbb{T}= & {\left[\begin{array}{lll}
x_{1} x_{2} & x_{1} y_{2} & x_{1} z_{2} \\
y_{1} x_{2} & y_{1} y_{2} & y_{1} z_{2} \\
z_{1} x_{2} & z_{1} y_{2} & z_{1} z_{2}
\end{array}\right] } \\
= & \frac{1}{3} \operatorname{Tr}(\mathbb{T}) \mathbb{1}+\frac{1}{2}\left[\begin{array}{ccc}
0 & x_{1} y_{2}-y_{1} x_{2} & x_{1} z_{2}-z_{1} x_{2} \\
y_{1} x_{2}-x_{1} y_{2} & 0 & y_{1} z_{2}-z_{1} y_{2} \\
z_{1} x_{2}-x_{1} z_{2} & z_{1} y_{2}-y_{1} z_{2} & 0
\end{array}\right] \\
& +\frac{1}{2}\left[\begin{array}{ccc}
x_{1} x_{2}+x_{2} x_{1}-\frac{2}{3} \operatorname{Tr}(\mathbb{T}) & x_{1} y_{2}+y_{1} x_{2} & x_{1} z_{2}+z_{1} x_{2} \\
y_{1} x_{2}+x_{1} y_{2} & y_{1} y_{2}+y_{2} y_{1}-\frac{2}{3} \operatorname{Tr}(\mathbb{T}) & y_{1} z_{2}+z_{1} y_{2} \\
z_{1} x_{2}+x_{1} z_{2} & z_{1} y_{2}-y_{1} z_{2} & z_{1} z_{2}+z_{2} z_{1}-\frac{2}{3} \operatorname{Tr}(\mathbb{T})
\end{array}\right] \\
= & \frac{1}{3} \operatorname{Tr}(\mathbb{T}) \mathbb{1}+\left[\begin{array}{ccc}
0 & c & b \\
-c & 0 & a \\
-b & -a & 0
\end{array}\right]+\left[\begin{array}{ccc}
D_{1} & A & B \\
A & D_{2} & C \\
B & C & D_{3}
\end{array}\right]
\end{aligned}
$$

with $D_{1}+D_{2}+D_{3}=0$.

Now, let's see how tensors transform. To this end, consider $\left|r^{\prime}\right\rangle=M|r\rangle$ the transformation of $|r\rangle$ by $M$. The corresponding dual transformation is: $\left\langle r^{\prime}\right|=\langle r| M^{\dagger}$, and then, the tensor transformation must be: $T_{x y}^{\prime}=\left|r^{\prime}\right\rangle\left\langle r^{\prime}|=M| r\right\rangle\langle r| M^{\dagger}=M T_{x y} M^{\dagger}$. Thus, while the coordinate transformation of a vector is computed as:

$$
\left(\begin{array}{l}
x^{\prime} \\
y^{\prime} \\
z^{\prime}
\end{array}\right)=(\mathbb{M})\left(\begin{array}{l}
x \\
y \\
z
\end{array}\right)
$$

\footnotetext{
${ }^{3}$ Please note that instead of $\mathbb{U}_{j}(\theta)=e^{i \frac{\theta}{2} \sigma_{j}}$ we can write the equivalent $\mathbb{U}_{j}(\theta)=e^{i \theta \hat{S}_{j}}$ equation, then, the relationship between the rotations generated by the orbital and spin angular momentum is complete.
} 
that of a tensor is calculated as follows:

$$
\left(\begin{array}{lll}
x_{1}^{\prime} x_{2}^{\prime} & x_{1}^{\prime} y_{2}^{\prime} & x_{1}^{\prime} z_{2}^{\prime} \\
y_{1}^{\prime} x_{2}^{\prime} & y_{1}^{\prime} y_{2}^{\prime} & y_{1}^{\prime} z_{2}^{\prime} \\
z_{1}^{\prime} x_{2}^{\prime} & z_{1}^{\prime} y_{2}^{\prime} & z_{1}^{\prime} z_{2}^{\prime}
\end{array}\right)=(\mathbb{M})\left(\begin{array}{lll}
x_{1} x_{2} & x_{1} y_{2} & x_{1} z_{2} \\
y_{1} x_{2} & y_{1} y_{2} & y_{1} z_{2} \\
z_{1} x_{2} & z_{1} y_{2} & z_{1} z_{2}
\end{array}\right)\left(\mathbb{M}^{\dagger}\right)
$$

In a similar way, let $|s\rangle=\left(\begin{array}{l}z_{1} \\ z_{2}\end{array}\right)$ and $\left|s^{\prime}\right\rangle=U|s\rangle$ be an arbitrary spinor and its $U$ transformation. We can build up a hermitian spinor by means the exterior product $|s\rangle\langle s|=\left(\begin{array}{c}z_{1} \\ z_{2}\end{array}\right)\left(\begin{array}{ll}z_{1}^{*} & z_{2}^{*}\end{array}\right)=\left(\begin{array}{ll}\left|z_{1}\right|^{2} & z_{1} z_{2}^{*} \\ z_{1}^{*} z_{2} & \left|z_{2}\right|^{2}\end{array}\right)$. Its coordinate transformation must be: $\left|s^{\prime}\right\rangle\left\langle s^{\prime}|=U| s\right\rangle\langle s| U^{\dagger}$.

As $\left|z_{1}\right|^{2},\left|z_{2}\right|^{2} \in \mathbb{R}$ and $\left(z_{1}^{*} z_{2}\right)^{*}=z_{1} z_{2}^{*}$, we can write $\left|z_{1}\right|^{2}=t+z,\left|z_{2}\right|^{2}=t-z, z_{1}^{*} z_{2}=x-i y, z_{1} z_{2}^{*}=x+i y$, with $x, y, z, t \in \mathbb{R}$, so that:

$$
|s\rangle\langle s|=\left(\begin{array}{cc}
t+z & x-i y \\
x+i y & t-z
\end{array}\right)=t \mathbb{1}+x \sigma_{x}+y \sigma_{y}+z \sigma_{z}
$$

We see then that a rank 2 hermitian spinor can be represented by a 2D hermitian matrix, not necessarily traceless (it will be traceless for $\mathrm{t}=0$ ), which in turn can always be expressed in terms of the identity matrix and the Pauli matrices (only in terms of Pauli's matrices if it is traceless).

\section{The experiment}

A $2 \pi$ rotation changes the sign of the spin function. However, wave functions' phases are not directly observable. The only way to detect them is to look at interference pattern of a rotated and a non-rotated state.

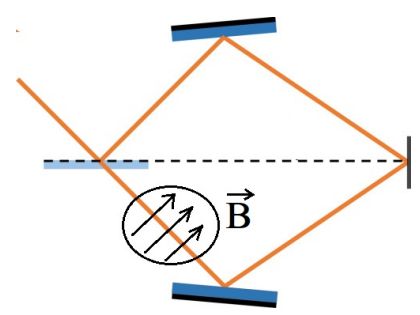

Then, we split a beam of neutrons ( paths, except that one of the branches crosses a region where a uniform magnetic field is applied. Then, strategically placed mirrors converge the two branches at the same point on a screen to record the interference pattern.

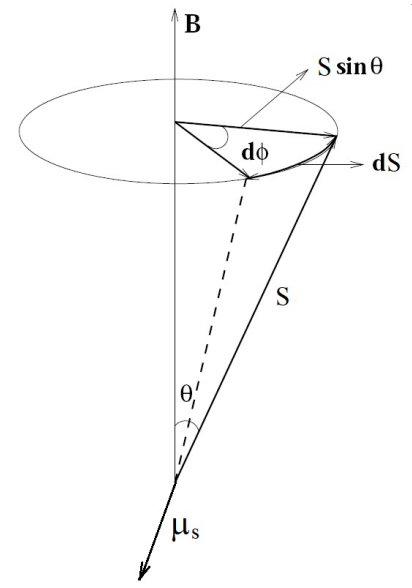


Associated with the spin angular momentum $|S\rangle$, neutrons have a dipole magnetic moment $\left|\mu_{s}\right\rangle=\beta|S\rangle$, with $\beta=\frac{e}{m}$. The magnetic field produces a torque $M$ on this dipole that rotates the spin $|S\rangle$ within the plane defined by the field $|B\rangle$ and the vector itself $|S\rangle$, thus generating an increase $d|S\rangle$ of the spin angular momentum perpendicularly to this plane: $M=\left|\mu_{s}\right\rangle \times|B\rangle=\frac{d|S\rangle}{d t}$. Then, $\frac{d|S\rangle}{d t}=\beta|S\rangle \times|B\rangle$.

As can be seen in the figure, $d|S\rangle=|S| \sin \theta d \phi|u\rangle$, where $|u\rangle$ is a unit vector along $d|S\rangle$. Then, $|S| \sin \theta \frac{d \phi}{d t}=\beta|S||B| \sin \theta \rightarrow \omega \equiv \frac{d \phi}{d t}=\beta B$, with $\omega$ the precession angular speed.

Neutrons crossing the field will change the spin phase. Should we call $\tau=\frac{2 \pi}{\omega}$ to the precession period then, the phase change period will be twice this period $\tau_{f}=\frac{4 \pi}{\omega}$. Therefore, if neutrons take a time $T$ to cross the magnetic field, the phase captured by the spin function will be $e^{i \omega T / 2}$.

The field strength $|B|$, i.e., the precession velocity $\omega$, modulates the interference pattern. We can calculate the difference of fields that produce two consecutive maximums in the interference pattern. For example, a maximum at $B=\omega=0$ will be followed by another at $\omega=4 \pi$. In 1975 , two research groups experimentally confirm this theoretical prediction.[10, 11]

We can now better understand the sentence in Le Bellac's book[3] (p. 234) saying that the actual identity rotation of an object in relation to its surroundings is not $2 \pi$ but $4 \pi$. The non-equivalence of $2 \pi$ and $4 \pi$ rotations can be displayed by rotating an object attached to an end of a ribbon with the other extreme fixed (Dirac's belt). As we rotate the object by an angle $2 \pi$ we produce a twist in the ribbon that there is no way to undo without further rotating the object. An additional $2 \pi$ rotation (up to $4 \pi$ ) produces an extra twist. However, if we move the object towards the fixed extreme of the ribbon and pass it underneath without rotations from right to left and then stretch the ribbon, the knot fades away and we return to the original position (see e.g. Staley[12]). The non-equivalence of $2 \pi$ and $4 \pi$ rotations is related to the two-fold connection of the $S O(3)$ group (while its covering group, $S U(2)$ is simply connected).

\section{1 $S U(2)$ and $S O(3)$ connection}

\subsection{1 $S U(2)$ connection}

$S U(2)$ elements are $2 \times 2$ unitary unimodular matrices $\mathbb{U}=\left(\begin{array}{cc}z_{1} & -z_{2}^{*} \\ z_{2} & z_{1}^{*}\end{array}\right)$ :

$$
\begin{gathered}
\mathbb{U} \mathbb{U}^{\dagger}=\left(\begin{array}{cc}
z_{1} & -z_{2}^{*} \\
z_{2} & z_{1}^{*}
\end{array}\right)\left(\begin{array}{cc}
z_{1}^{*} & -z_{2}^{*} \\
-z_{2} & z_{1}
\end{array}\right)=\left(\begin{array}{cc}
\left|z_{1}\right|^{2}+\left|z_{2}\right|^{2} & z_{1} z_{2}^{*}-z_{2}^{*} z_{1} \\
z_{2} z_{1}^{*}-z_{1}^{*} z_{2} & \left|z_{1}\right|^{2}+\left|z_{2}\right|^{2}
\end{array}\right)=\left(\begin{array}{cc}
1 & 0 \\
0 & 1
\end{array}\right) \\
\operatorname{det} \mathbb{U}=\left|z_{1}\right|^{2}+\left|z_{2}\right|^{2}=1
\end{gathered}
$$

Should we write $z_{1}=x_{1}+i x_{2}, z_{2}=x_{3}+i x_{4}$ then the $\left|z_{1}\right|^{2}+\left|z_{2}\right|^{2}=1$ condition translates into: $x_{1}^{2}+x_{2}^{2}+x_{3}^{2}+x_{4}^{2}=1$. This is the equation defining a unit radius $S^{3}$ sphere embedded in $\mathbb{R}^{4}$. Then, we can set a differentiable isomorphism $S U(2) \leftrightarrow S^{3} \subset \mathbb{R}^{4}$. Since spheres are simply connected so must be $S U(2)$.

\subsection{2 $S O(3)$ is doubly connected}

Any rotation can be defined by a vector of length equal to the angle of rotation and direction $(\theta, \phi)$ that of the rotation axis: $R_{\mathbf{n}}(\psi)=R(\psi, \theta, \phi), 0 \leq \theta \leq \pi, 0 \leq \phi \leq 2 \pi$. Since $R_{-\mathbf{n}}(\psi)=R_{\mathbf{n}}(\psi)$ we can circumscribe $\psi: 0 \leq \psi \leq \pi$.

We can represent the parameters of any rotation $R$ by the points of a radius $\pi$ sphere, with the redundancy that two antipodal points (i.e., two points on the surface that are the two extremes of a diameter) are the same point, as the rotations $\pi$ and $-\pi$ about any axis are the same rotation.[13] This antipodal 
identification leads the images of two rotations $R_{1}$ and $R_{2}$ in the parameter space to join by two nonequivalent paths (see figure).

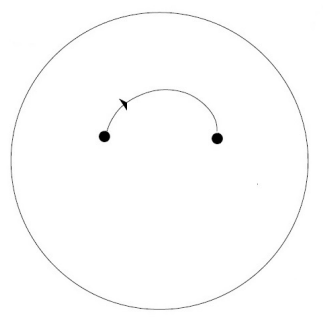

(a)

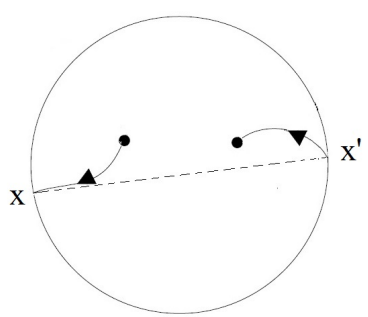

(b)

Path (b) cannot be continuously deformed into path (a), because if we displace $x$ on the surface, $x$ ' automatically rush to get the antipodal position, so they never can melt yielding path (a). Additionally, should we move $x$ from the surface, then $x^{\prime}$ disappears and the path breaks into two separate paths.

Also, starting from a zero rotation (central point of the sphere) and rotating $2 \pi$ about e.g. the vertical axis (we start from the center, go to the north pole, jump to the south pole and return to the center) the path cannot be continuously deformed and finally melt into a single point at the sphere center .... but a $4 \pi$ rotation does allow it! (see figure).

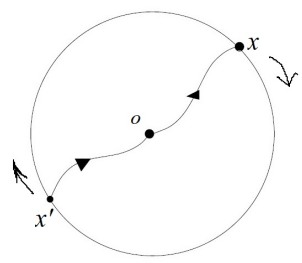

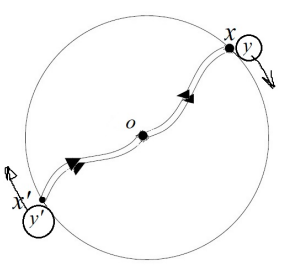
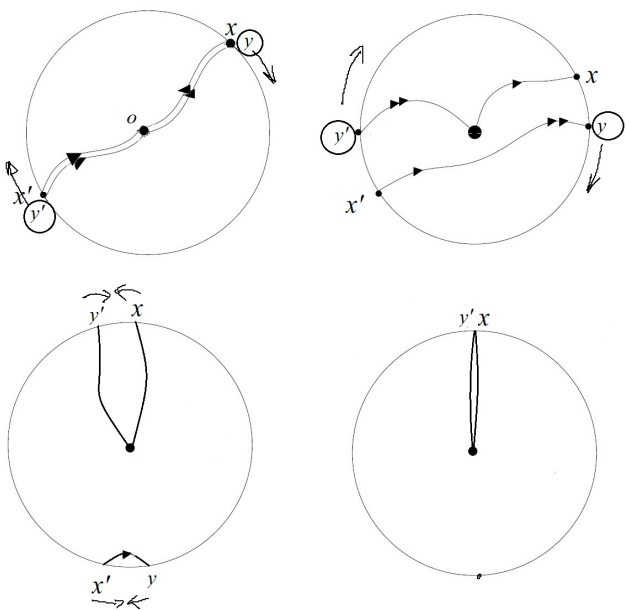

Let's come back to the Dirac' belt: a vector (or an object) is attached to the extreme of a ribbon, the other extreme being fixed, and rotate the vector. Then, the ribbon accumulates rotations as twists. After a $2 \pi$ rotation the vector recover its original position but the ribbon remains twisted. If we do not allow rotations on the ribbon extremes, i.e. do not allow rotations of the original and rotated vectors attached to both ribbon extremes, there is no way to undo the intermediate rotations that keep the ribbon twisted. On the other hand, after a $4 \pi$ rotation there is a way to undo the intermediate rotations and smooth the ribbon while forbidding rotations of the original and rotated vectors. This fact harmonizes with the concept of rotation it in relation to the surroundings of Le Bellac's book.[3] 

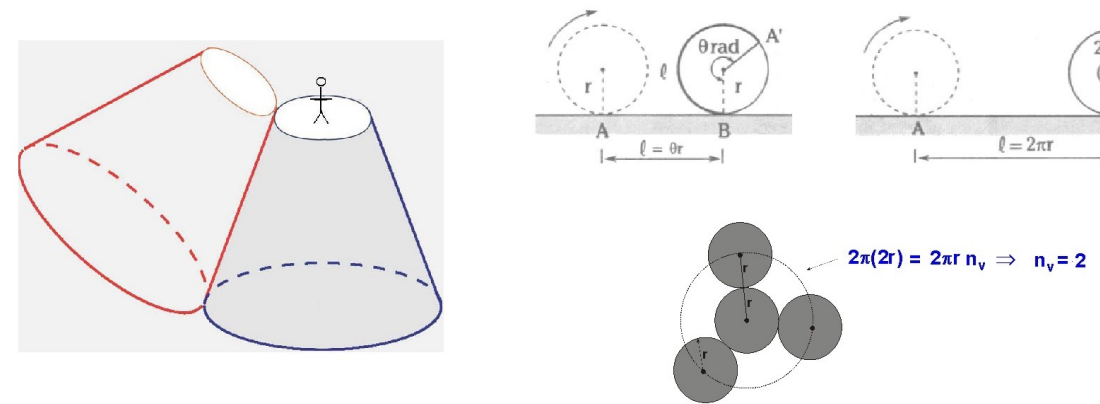

There is another trick, called the Weyl double cone, which even better exemplifies the concept of rotation in relation to the surroundings. Weyl's trick is akin to the rotation of a two coins: a coin rotates around another alike that is kept fixed. We can see in the figure that two complete turns of the rotating coin are needed to return to the starting point (when a coin makes a complete round on a straight line, its center moves a distance $2 \pi R$, with $R$ the coin radius and also the rotation radius, while the rotation radius is $2 R$-sum of the radii of the two coins- when a coin rotates around another. So, two rotations $2 \times 2 \pi R$ are needed to advance the length $2 \pi(2 R))$.

But attention, should we rotate a radius $R$ coin about another having a radius $2 R$, then the rotation radius is $3 R$, which means that only after three complete turns can we recover the original position. A $1.75 R$ radius of the inner coin yields a $2.75 R$ rotation radius so we need 4 full turns around the central coin to return to the starting position (meanwhile the mobile coin rotates 11 times around its center). Therefore, while the Dirac's belt and Weyl's cones are ingenious tricks, they should not be considered as any proof of the spin functions' properties.

\section{Appendix: The set of wave functions must build basis for the symmetry group representations}

We include here an elementary argument supporting Pauli's suggestion on the requirement that the set of eigenfunctions should build basis for the symmetry group representations. To this end let $R_{k}$ be any transformation leaving invariant the Hamiltonian $\hat{H}: R_{k} \hat{H} R_{k}^{-1}=\hat{H}$ and $\Psi_{i}, E_{i}$ and eigenfuction and the associated eigenvalue. Let $\Phi_{k}=R_{k} \Psi_{i}$. It is straightforward to show that $\Phi_{k}$ is an eigenfunction of $\hat{H}$ associated to the same eigenvalue $E_{i}$ :

$$
\hat{H} \Phi_{k}=R_{k} \hat{H} R_{k}^{-1} \Phi_{k}=R_{k} \hat{H} R_{k}^{-1} R_{k} \Psi_{i}=R_{k} \hat{H} \Psi_{i}=R_{k} E_{i} \Psi_{i}=E_{i} R_{k} \Psi_{i}=E_{i} \Phi_{k}
$$

This holds for every $R_{k}$ belonging the group of transformations $G$ leaving invariant the Hamiltonian. The set of degenerate eigenfunctions $W=\left\{\Phi_{k}=R_{k} \Psi_{i}, \forall k\right\}$ expand a linear space stable under $G$, i.e., any $R_{k} \in G$ acting upon a function of this space yields another function within this space, i.e. an $\hat{H}$ eigenfuction associated to the eigenvalue $E_{i}$. We say then that $W$ form a basis for a representation of the group $G$. Is this an irreducible representation? The answer is yes. One can try to bring about e.g. the contra-example of hydrogen atom and the rotation group in three dimensions $O(3)$, where e.g. the $2 s$ orbital does not belong to the same irreducible representation than the three $2 p$ orbitals while being $2 s$ and $2 p$ degenerated. However, $O(3)$ is not the group of transformations $G$ leaving invariant the Hamiltonian of the hydrogen atom but just the space or geometrical symmetry group of it. In addition to the geometric transformations, the hydrogen atom Hamiltonian is also invariant under transformations involving simultaneously coordinates and momenta. The set of all transformations leaving invariant the hydrogen atom Hamiltonian form (or is isomorphic to) the hydrogen atom dynamical symmetry group $O(4) \cdot[13]$ 


\section{References}

[1] R. McWeeny, Quantum Mechanics: principles and formalism, Pergamon Press, Oxford 1972.

[2] E. Merzbacher, Am. J. Phys. 30 (1962) 237.

[3] M. Le Bellac, Quantum Physics, Cambridge University Press, 2006.

[4] J. Riess, Helv. Phys. Acta 45 (1972) 1066.

[5] https ://en.wikipedia.org/wiki/Elliptic_partial_differential_equation

[6] V.B. Ho and M.J. Morgan, J. Phys. A: Math and Gen. 29 (1996)1497.

[7] veure pag. 201 en J.J. Sakurai, Modern Quantum Mechanics Addison-Wesley 1994.

[8] veure pag. 138 en Frank F. Pilar, Elementary Quantum Chemistry, McGraw-Hill, NY 1968.

[9] A. M. Steane, arXiv 1312.3824 (2013) (http ://arxiv.org/abs/1312.3824v1)

[10] H. Rauch, A. Zeilinger, G. Badurek, A. Wilfing, W. Bauspiess and U. Bonse, Phys. Lett. A 54 (1975) 425

[11] S. A. Werner, R. Colella, A. W. Overhauser, and C. F. Eagen, Phys. Rev. Lett. 35 (1975) 1053.

[12] M. Staley, Eur. J. Phys. 31 (2010) 467.

[13] A. W. Joshi, Elements of Group Theory for Physicists, Wiley Eastern, New Delhi 1982. 